Pacific Journal of Mathematics

A THEOREM ON RINGS OF OPERATORS 


\title{
A THEOREM ON RINGS OF OPERATORS
}

\author{
IRVING KA PLANSKY
}

1. Introduction. The main result (Theorem 1) proved in this paper arose in connection with investigations on the structure of rings of operators. Because of its possible independent interest, it is being published separately.

The proof of Theorem 1 is closely modeled on the discussion in Chapter I of [3]. The connection can be briefly explained as follows. Let $N$ be a factor of type $\mathrm{II}_{1}$; then in addition to the usual topologies on $N$, we have the metric defined by $[[A]]^{2}=T\left(A^{*} A\right), T$ being the trace on $N$. Now it is a fact that in any bounded subset of $N$, the [[ ]]-metric coincides with the strong topology-this is the substance of Lemma 1.3.2 of [3]. In the light of this observation, it can be seen that Theorem 1 is essentially a generalization (to arbitrary rings of operators) of the ideas in Chapter I of [3].

Before stating Theorem 1, we collect some definitions for the reader's convenience. Let $R$ be the algebra of all bounded operators on a Hilbert space $H$ (of any dimension). In $R$ we have a natural norm and *-operation. A typical neighborhood of 0 for the strong topology in $R$ is given by specifying $\epsilon>0, \xi_{1}, \cdots$, $\xi_{n} \in H$, and taking the set of all $A$ in $R$ with $\left\|A \xi_{i}\right\|<\epsilon$; for the weak topology we specify further vectors $\eta_{1}, \cdots, \eta_{n} \in H$ and take the set of all $A$ with $\left|\left(A \xi_{i}, \eta_{i}\right)\right|<\epsilon$. By a *-algebra of operators we mean a self-adjoint subalgebra of $R$, that is, one containing $A^{*}$ whenever it contains $A$; unless explicitly stated, it is not assumed to be closed in any particular topology. For convex subsets of $R$, and in particular for subalgebras, strong and weak closure coincide [2, Th. 5]. An operator $A$ is self-adjoint if $A^{*}=A$, normal if $A A^{*}=A^{*} A$, unitary if $A A^{*}$ $=A^{*} A=$ the identity operator $I$.

2. The main result. We shall establish the following result.

THE OREM 1. Let $M, N$ be *algebras of operators on Hilbert space, $M \subset N$, and suppose $M$ is strongly dense in $N$. Then the unit sphere of $M$ is strongly dense in the unit sphere of $N$.

Received October 6,1950. This paper was written in connection with a research project on spectral theory, sponsored by the Office of Naval Research.

Pacific J. Math. 1 (1951), 227-232. 
We shall break up the early part of the proof into a sequence of lemmas. Lemma 1 is well known and is included only for completeness.

LEMMA 1 . In the unit sphere of $R$, multiplication is strongly continuous, jointly in its variables; and any polynomial in $n$ variables is strongly continuous, jointly in its arguments.

Proof. It is easy to see that multiplication is strongly continuous separately in its variables, even in all of $R$. Consequently $[1$, p.49] we need only check the continuity of $A B$ at $A=B=0$. Since $\|A\| \leq 1$, this is a consequence of

$$
\|A B \xi\| \leq\|A\|\|B \xi\| \leq\|B \xi\| \text {. }
$$

Since addition and scalar multiplication are continuous (in all of $R$ ), the continuity of polynomials follows.

The precaution taken in the next lemma, in defining the mapping on the pair $\left(A, A^{*}\right)$, is necessary since $A \longrightarrow A^{*}$ is not strongly continuous.

Lemma 2. Let $f(z)$ be a continuous complex-valued function, defined for $|z| \leq 1$. Then the mapping $\left(A, A^{*}\right) \rightarrow f(A)$ is strongly continuous on the normal operators of the unit sphere of $R$.

Proof. We are given a normal operator $A_{0}$ with $\left\|A_{0}\right\| \leq 1$, a positive $\epsilon$, and vectors $\xi_{i}$ in $H(i=1, \cdots, n)$. We have to show that by taking $A, A^{*}$ to be normal with norm $\leq 1$, and in suitable strong neighborhoods of $A_{0}, A_{0}^{*}$, we can achieve

$$
\left\|\left[f(A)-f\left(A_{0}\right)\right] \xi_{i}\right\|<\epsilon .
$$

By the Weierstrass approximation theorem, there exists a polynomial $g$ in two variables such that

$$
\left|g\left(z, z^{*}\right)-f(z)\right|<\epsilon / 3,
$$

for $|z| \leq 1, z^{*}$ denoting the conjugate complex of $z$. By elementary properties of the functional calculus for normal operators, we deduce from (2):

$$
\begin{gathered}
\left\|g\left(A, A^{*}\right)-f(A)\right\|<\epsilon / 3, \\
\left\|g\left(A_{0}, A_{0}^{*}\right)-f\left(A_{0}\right)\right\|<\epsilon / 3 .
\end{gathered}
$$

By Lemma 1 , if we take $A, A^{*}$ in appropriate neighborhoods of $A_{0}, A_{0}^{*}$, we have 


$$
\left\|\left[g\left(A, A^{*}\right)-g\left(A_{0}, A_{0}^{*}\right)\right] \xi_{i}\right\|<\epsilon / 3 .
$$

By combining (3), (4), and (5) we obtain (1).

The next lemma follows from Lemma 2 as soon as it is admitted that $*$ is strongly continuous on unitary operators. This can, for example, be deduced from two known facts: (a) the strong and weak topologies coincide on the set of unitary operators, and $(\mathrm{b}) *$ is weakly continuous.

LEMMA 3. Let $f$ be a continuous complex-valued function defined on the circumference of the unit circle. Then the mapping $U \rightarrow f(U)$ is strongly continuous on the set of unitary operators.

The Cayley transform is the mapping $A \rightarrow(A-i)(A+i)^{-1}$; it is defined for any self-adjoint operator and sends it into a unitary operator.

LEMMA 4. The Cayley transform is strongly continuous on the set of all selfadjoint operators.

Proof. We have the identity

$$
(A-i)(A+i)^{-1}-\left(A_{0}-i\right)\left(A_{0}+i\right)^{-1}=2 i(A+i)^{-1}\left(A-A_{0}\right)\left(A_{0}+i\right)^{-1} .
$$

When $A$ is self-adjoint, we have $\left\|(A+i)^{-1}\right\| \leq 1$. In order to make the left side of (6) small on a vector $\xi$, it therefore suffices to make $A-A_{0}$ small on the vector $\left(A_{0}+i\right)^{-1} \xi$.

We shall prove a stronger form of Lemma 5 below (Corollary to Theorem 2).

LEMMA 5. Let $h$ be a real-valued function defined on the real line, and suppose that $h$ is continuous and vanishes at infinity. Then the mapping $A \rightarrow h(A)$ is strongly continuous on the set of all self-adjoint operators.

Proof. Define

$$
\begin{array}{rlrl}
f(z) & =h\left[-i(z+1)(z-1)^{-1}\right] & \text { for }|z|=1, & z \neq 1, \\
& =0 & \text { for } z=1 .
\end{array}
$$

Then $f$ is continuous on the circumference of the unit circle. Moreover,

$$
h(A)=f\left[(A-i)(A+i)^{-1}\right] \text {. }
$$

The mapping $A \longrightarrow h(A)$ is thus the composite of two maps: the Cayley transform, 
and the mapping on unitary operators given by $f$. By Lemmas 4 and 3 , these latter two maps are strongly continuous. Hence so is $A \longrightarrow h(A)$.

Proof of Theorem 1. There is clearly no loss of generality in assuming $M$ and $N$ to be uniformly closed, for the unit sphere of $M$ is even uniformly dense in the unit sphere of its uniform closure.

Let us write $Z$ for the set of self-adjoint elements in $M$, and $Z_{1}$ for the unit sphere of $Z$. Let $B$ be a given self-adjoint element in $N,\|B\| \leq 1$. By hypothesis, $B$ is in the strong closure of $M$. We shall argue in two successive steps that $B$ is actually in the strong closure of $Z_{1}$. We begin by remarking that $B$ is in the weak closure of $M$, since the latter coincides with the strong closure of $M$. Now * is weakly continuous, and hence so is the mapping $A \rightarrow\left(A+A^{*}\right) / 2$. This mapping leaves $B$ fixed, and sends $M$ onto $Z$; hence $B$ is in the weak closure of $Z$. Since $Z$ is convex, this coincides with the strong closure of $Z$.

Let $h(t)$ be any real-valued function of the real variable $t$ which is continuous and vanishes at infinity, satisfies $|h(t)| \leq 1$ for all $t$, and satisfies $h(t)=t$ for $|t| \leq 1$. We have that $h(B)=B$. Also $h$ can be meaningfully applied within $Z$, since we have assumed $M$ to be uniformly closed, and in fact $h(Z)=Z_{1}$. By Lemma 5 , the mapping $A \longrightarrow h(A)$ is strongly continuous on self-adjoint operators. Hence $B$ is in the strong closure of $Z_{1}$.

This accomplishes our objective as far as self-adjoint operators are concerned. To make the trahsition to an arbitrary operator, we adopt the device of passing to a matrix algebra. ${ }^{1}$ Let $N_{2}$ be the algebra of two-by-two matrices over $N$. In a natural way, $N_{2}$ is again a uniformly closed *algebra of operators on a suitable Hilbert space (compare $\$ 2.4$ of [3]). It contains in a natural way $M_{2}$, the two-bytwo matrix algebra over $M$. The strong topology on $N_{2}$ is simply the Cartesian product of the strong topology for the four replicas of $N$; thus $M_{2}$ is again strongly dense in $N_{2}$. Now let $C$ be any operator in $N,\|C\| \leq 1$. We form

$$
D=\left(\begin{array}{ll}
0 & C \\
C^{*} & 0
\end{array}\right)
$$

and we note that $D \in N_{2}, D^{*}=D,\|D\| \leq 1$. Let $U$ be any proposed strong neighborhood of $D$. By what we have proved above, there exists in $U$ a self-adjoint element $F$,

${ }^{1} \mathrm{I}$ am indebted to $\mathrm{P}$. R. Halmos for this device, which considerably shortened my original proof of Theorem 1 . 


$$
F=\left(\begin{array}{ll}
G & H \\
H^{*} & K
\end{array}\right)
$$

with $F \in M_{2},\|F\| \leq 1$. By suitable choice of $U$ we can make $H$ lie in a given strong neighborhood of $C$. Also $\|F\| \leq 1$ implies $\|H\| \leq 1$. This proves that $C$ lies in the strong closure of the unit sphere of $M$, and concludes the proof of Theorem 1.

3. Remarks. (a) Since strong and weak closure coincide for convex sets, we can, in the statement of Theorem 1, replace "strongly" by "weakly" at will.

(b) From Theorem 1 we can deduce that portion of $[2, T h .8]$ that asserts that a *-algebra of operators is strongly closed if its unit sphere is strongly closed; but it does not appear to be possible to reverse the reasoning.

(c) As Dixmier has remarked [2, p.399], Theorem 1 fails if $M$ is merely assumed to be a subspace (instead of a *-subalgebra).

4. Another result. In concluding the paper we shall return to Lemma 5 and show that the hypothesis can be weakened to the assumption that $h$ is bounded and continuous. It should be noted that we cannot drop the word "bounded," since for example it is known that the mapping $A \rightarrow A^{2}$ is not strongly continuous.

Actually we shall prove a still more general result, which may be regarded as a generalization of Lemma 4.2.1 of [3].

THEOREM 2. Let $h(t)$ be a bounded real-valued Baire function of the real variable $t$, and $A_{0}$ a self-adjoint operator. Let $S$ be the spectrum of $A_{0}$, and $T$ the closure of the set of points at which $h$ is discontinuous; suppose $S$ and $T$ are dis joint. Then the mapping on self-adjoint operators, defined by $A \rightarrow h(A)$, is continuous at $A=A_{0}$.

Proof. We may suppose that

$$
|h(t)| \leq 1
$$

for all $t$. Given $\epsilon>0$, and vectors $\xi_{i}$, we have to show that for $A$ in a suitable strong neighborhood of $A_{0}$, we have

$$
\left\|\left[h(A)-h\left(A_{0}\right)\right] \xi_{i}\right\|<\epsilon .
$$

Choose a function $k(t)$ which satisfies: (a) $k$ is continuous and vanishes at infinity, (b) $k(t)=1$ for $t$ in $S$, (c) $k(t)=0$ for $t$ in an open set containing $T$. Define 
$p=h k, q=1-k+h k$. Then $p=q=h$ on $S$, and so

$$
p\left(A_{0}\right)=q\left(A_{0}\right)=h\left(A_{0}\right) .
$$

Also $p$ and $q-1$ are continuous and vanish at infinity. Hence Lemma 5 is applicable, and for a certain strong neighborhood of $A_{0}$ we have

$$
\left\|\left[p(A)-p\left(A_{0}\right)\right] \xi_{i}\right\|<\epsilon / 4, \quad\left\|\left[q(A)-q\left(A_{0}\right)\right] \xi_{i}\right\|<\epsilon / 2 .
$$

The following is an identity:

$$
h=(1-h) p+h q \text {. }
$$

From (9) and (11) we get

$$
h(A)-h\left(A_{0}\right)=[1-h(A)]\left[p(A)-p\left(A_{0}\right)\right]+h(A)\left[q(A)-q\left(A_{0}\right)\right] .
$$

From (7), (10), and (12), we deduce (8), as desired.

If in particular $h$ is continuous, then $T$ is void and we get a simplified corollary.

COROLLARY. Let $h(t)$ be a continuous bounded real-valued function of the real variable . Then the mapping $A \longrightarrow h(A)$ is strongly continuous on the set of all self-adjoint operators.

\section{REFERENCES}

1. N. Bourbaki, Éléments de Mathématique, Livre III, Topologie Générale, Actualités Sci. Ind., No.916, Paris, 1942.

2. J. Dixmier, Les fonctionelles linéaires sur l'ensemble des opérateurs bornés d'un espace de Hilbert, Ann. of Math. 51 (1950), 387-408.

3. F. J. Murray and J. von Neumann, On rings of operators IV, Ann. of Math. 44 (1943), 716-808.

University of Chichao 


\title{
PACIFIC JOURNAL OF MATHEMATICS
}

\section{EDITORS}

\author{
Herbert BuSEMANN \\ R. M. RoBINSON \\ University of Southern California \\ University of California \\ Los Angeles 7, California \\ Berkeley 4, California \\ E. F. BEC KENBACH, Managing Editor \\ University of California \\ Los Angeles 24, California
}

\section{ASSOCIATE EDITORS}
R. P. DILWORTH
P. R. HALMOS
BØRGE JESSEN
J. J. STOKER
HERBERT FEDERER
HEINZ HOPF
PAUL LÉVY
MARSHALL HALL
R. D. JAMES
GEORGE PÓLYA
E. G. STRAUS
KÖSAKU YOSIDA

\section{SPONSORS}

UNIVERSITY OF BRITISH COLUMBIA

CALIFORNIA INSTITUTE OF TECHNOLOGY

UNIVERSITY OF CALIFORNIA, BERKELEY

UNIVERSITY OF CALIFORNIA, DAVIS

UNIVERSITY OF CALIFORNIA, LOS ANGELES

UNIVERSITY OF CALIFORNIA, SANTA BARBARA

OREGON STATE COLLEGE

UNIVERSITY OF OREGON
UNIVERSITY OF SOUTHERN CALIFORNIA

STANFORD UNIVERSITY

WASHINGTON STATE COLLEGE

UNIVERSITY OF WASHINGTON

AMERICAN MATHEMATICAL SOCIETY

NATIONAL BUREAU OF STANDARDS, INSTITUTE FOR NUMERIGAL ANALYSIS

Mathematical papers intended for publication in the Pacific Journal of Mathematics should be typewritten (double spaced), and the author should keep a complete copy. Manuscripts may be sent to any of the editors. All other communications to the editors should be addressed to the managing editor, E. F. Beckenbach, at the address given above.

Authors are entitled to receive 100 free reprints of their published papers and may obtain additional copies at cost.

The Pacific Journal of Mathematics is published quarterly, in March, June, September, and December. The price per volume (4 numbers) is $\$ 8.00$; single issues, $\$ 2.50$. Spécial price to individual faculty members of supporting institutions and to members of the American Mathematical Society: $\$ 4.00$ per volume; single issues, $\$ 1.25$.

Subscriptions, orders for back numbers, and changes of address should be sent to the publishers, University of California Press, Berkeley 4, California.

UNIVERSITY OF CALIFORNIA PRESS - BERKELEY AND LOS ANGELES 


\section{Pacific Journal of Mathematics}

\section{Vol. 1, No. $2 \quad$ December, 1951}

Tom M. (Mike) Apostol, On the Lerch zeta function ................. 161

Ross A. Beaumont and Herbert S. Zuckerman, A characterization of the subgroups of the additive rationals ....................... 169

Richard Bellman and Theodore Edward Harris, Recurrence times for the Ehrenfest model................................... 179

Stephen P.L. Diliberto and Ernst Gabor Straus, On the approximation of a function of several variables by the sum of functions of fewer

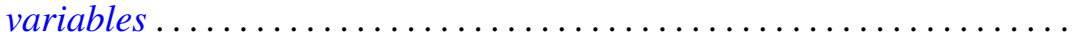

Isidore Isaac Hirschman, Jr. and D. V. Widder, Convolution transforms with complex kernels ................................ 211

Irving Kaplansky, A theorem on rings of operators .............. 227

W. Karush, An iterative method for finding characteristic vectors of a symmetric matrix............................... 233

Henry B. Mann, On the number of integers in the sum of two sets of positive integers ......................................... 249

William H. Mills, A theorem on the representation theory of Jordan

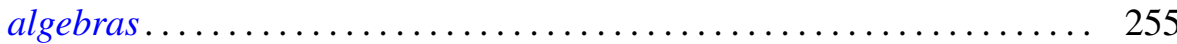

Tibor Radó, An approach to singular homology theory.............. 265

Otto Szász, On some trigonometric transforms ................... 291

James G. Wendel, On isometric isomorphism of group algebras ......... 305

George Milton Wing, On the $L^{p}$ theory of Hankel transforms ... 